\title{
Ritualpflanzen bei schamanistischen Krankenheilungs-Ritualen der Benuaq Dayak in Ost-Kalimantan (Borneo)
}

HERWIG ZAHORKA

\begin{abstract}
The Dayak Benuaq people believe in many and diverse terrestrial ghosts or spirits which exist in the environment. Some of them are believed to have the power to remove the soul of a human body part or organ and, at the same time, implant disease. As a consequence, the part of the body part or organ gets sick. The mission of the shamans is to identify the ghost or spirit responsible during nocturnal rituals and to submit all of the diverse ritual offerings possible, including making an exchange of a soul made from ironwood (Eusideroxylon zwageri). The captured soul is massaged into the sick body part of the patient. To perform this ritual, a great number of traditional and institutionalized plants are essential. White rice and rice colored with black, red, yellow and green dyes plays an important role in attracting and satisfying the ghosts.
\end{abstract}

\section{Zusammenfassung}

In der Vorstellung der Benuaq Dayak können einige der vielen und verschiedenen Naturgeister eine menschliche Körperteilseele rauben und dabei einen Krankheitsstoff hinterlassen. Als Folge erkrankt dieses Organ. Die Aufgabe der Schamanen ist es, den krankmachenden Geist zu identifizieren und ihm die diversen, rituellen Opfergaben und eine Tauschseele aus Eisenholz (Eusideroxylon zwageri) anzubieten. Der Krankheitsstoff kann vom Schamanen mit einem fein gespleißten Bananenblatt aus dem erkrankten Körperteil extrahiert und einer geschnitzten Figur übergeben oder in das Blut der Opfertiere geschüttet werden. Die eingefangene Seele massiert der Schamane in den erkrankten Körperteil des Patienten ein. Für das Ritual ist eine sehr große Zahl traditionell festgelegter Pflanzen erforderlich. Reis in den Farben Schwarz, Rot, Gelb, Grün und Weiß lockt die Geister heran und befriedigt sie.

\section{Grundvorstellung von Krankheit und Heilungsbedingungen}

In der überlieferten Vorstellung der hier zitierten Ohong Benuaq Dayak, die zur Luangan-Gruppe gehören und in den Langhausdörfern Pentat, Lempunah, Muara Nayan, Mancong und Keranau entlang des Ohong-Flusses leben, hat ein lebender Mensch neben seiner Geistseele auch sieben Körperteilseelen. Verschiedene Naturgeister sind in der Lage, aus bestimmten Gründen eine derartige Seele zu rauben und an ihrer Stelle einen Krankheitsstoff zu hinterlassen. Am häufigsten betroffen sind hierbei die Bauch-, Kopf- und Herzseele. Als Folge erkranken die entsprechenden Organe.

Heilungsbemühungen können nur erfolgreich sein, wenn der Krankheitsstoff aus dem erkrankten Körperteil entfernt und die vermisste Seele wieder eingesetzt wird. Dies ist Aufgabe der als Medium zur Geisterwelt agierenden Schamanen, die in nächtlichen Ritualen den krankmachenden Geist identifizieren und ihm diverse, meist pflanzliche, Opfergaben anbieten müssen. Ist der Geist willig, so kann ihm der Krankheitsstoff zurückgegeben werden, und der Schamane erfährt im Trancezustand, wo er die fehlende Körperteilseele des Patienten finden kann. Für dieses Krankenheilungsritual, das meist mit Steigerungen vier Nächte lang durchgeführt wird, ist eine große Zahl bestimmter Pflanzenarten erforderlich. Dabei werden bis zu 59 Arten aus 32 Familien, darunter viele Medizinalpflanzen, verwendet (GöNNER 2002).

\section{Altar und Selbstschutzpflanzen des Scha- manen}

Zur Durchführung des Heilungsrituals ist ein Altar mit verschiedenen Aufbauten erforderlich. Davor stehen zwei abgedeckte antike chinesische Vorratsvasen und ein großer Gong. Der quadratische, erhöhte Altar ist aus dem ,geisterrelevanten" Holz von Melicope incana (Rutaceae) hergestellt. Er ist von acht Arten umgeben: Musa paradisiaca (Banane), Areca catechu (Betelpalme), Costus spec. (Schraubeningwer), Caryota mitis (Fischschwanzpalme), Cordyline fruticosa (Keulenlilie), Eusideroxylon zwageri (Eisenholz, Lauraceae), Melicope incana $=$ Euodia alba (Stinkesche), Macaranga triloba (Euphorbiaceae).

Diese Arten stehen in Beziehung zu verschiedenen Geisterfamilien, sie werden immer bei rituellen Handlungen benutzt und auch zu Ritualgegenständen verarbeitet. Das alkaloidreiche Melicope ist das „Geisterholz“. Eusideroxy- 
lon hat mystische Kräfte und kann hilfreiche Geister heranholen. Dem Schamanen muss es gelingen, die Familien der Baum-, Wald-, Feldund Wassergeister in den Raum zu locken, damit sie die Opfergaben annehmen können. Sie werden sich dann auf den Zweigen der Bäume oder in kleinen Häuschen niederlassen. Schüsseln mit den Opfergaben sind mit kronenförmigen Kuppeln aus jungen Blättern der Kokospalme verziert. Auch die Fransenwände sind daraus gefertigt. Die zur Abschreckung böser Geiste angebrachten rot und gelb eingefärbten Fransen sind aus den Blättern der Licuala-Palme gefertigt. Weitere Zierfransen zum Anlocken von Geistern stammen von jungen Blätter der Arenga pinnata.

Der Schamane beginnt die Sitzung mit einem scharfen Pfiff auf einem ausgehöhlten Eckzahn des Malayenbärs, um die Geister aufmerksam zu machen. Um sich selbst vor böswilligen Geistern zu schützen, bestreicht er sich mit weißer Reispaste und besprengt sich und alle Anwesenden mit dem Saft aus den Blüten der Betelpalme. Dafür benutzt er ein Zweiglein von Basilikum. Dieses „Weihwasser“-Ritual dürfte ein alt-hinduistisches Erbe aus der Zeit des Hindu-Reichs von König Mulawarman sein, das in diesem Gebiet bis zur Ankunft des Islam existierte und schon um 400 v. Chr. die ersten Schrifttafeln im Bereich des heutigen Indonesien mit Lettern in Sanskrit produzierte. Diese Vermutung dürfte auch auf die Verwendung von „Weihrauch“ zu Beginn dieses Rituals zutreffen. Dazu werden Stückchen der Wurzelknollen von Dianella ensifolia in ein Schüsselchen mit glühender Holzkohle geworfen. Die Pflanze enthält ein natürliches Insektizid. Schließlich wirft der Schamane dreimal heiligen, gelben Reis für die Reisgöttin über seine Schulter in Richtung Ein-

Abb. 1 (oben): Der rituelle Altar mit Opfergaben ist von acht Ritualpflanzen umgeben. Links das heilige Tuch mit herabhängenden jungen Blütenständen der Betelpalme.

Abb. 2 (Mitte): Der Schamane umtanzt mit seinem Blattschwert das heilige Tuch, seine Verbindung zur Geisterwelt.

Abb. 3 (unten): Beten, um eine Erleuchtung zur Identifizierung des Schadgeistes zu erhalten.
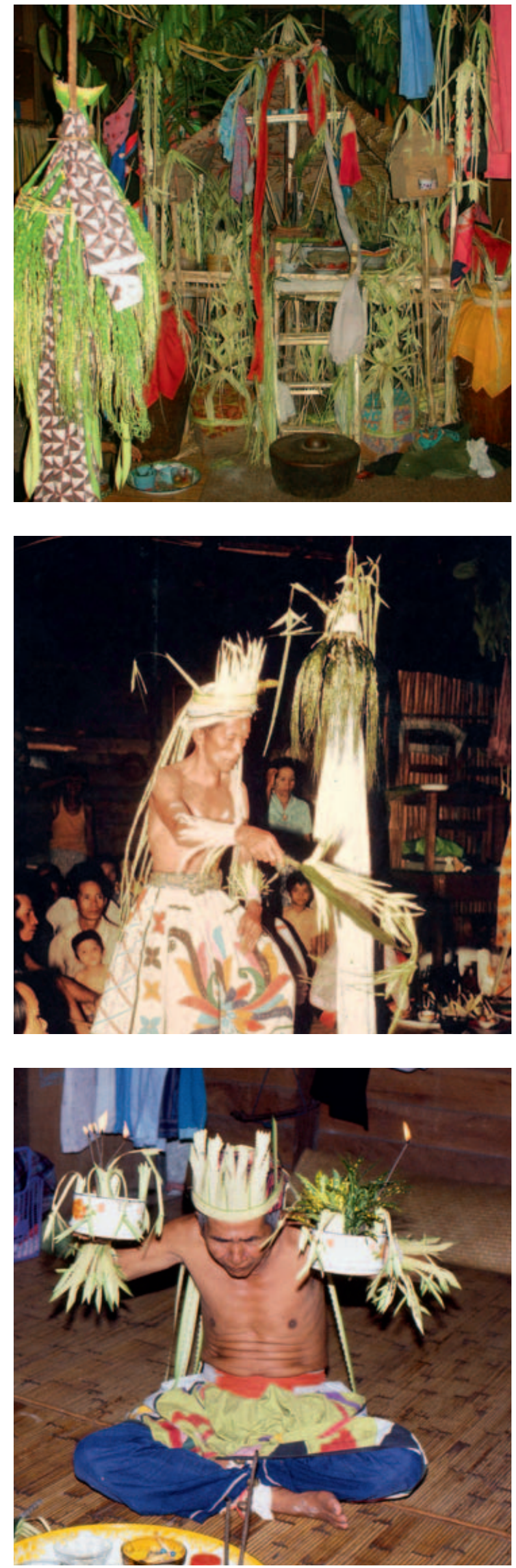

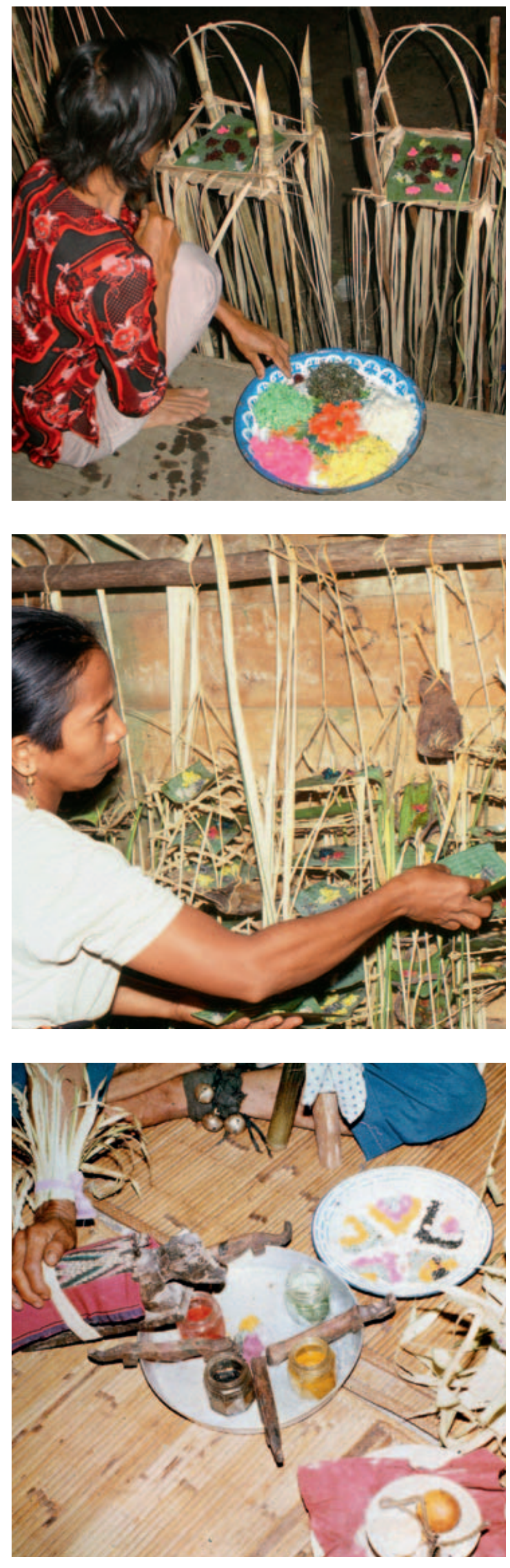

gang. Gelb gefärbt wird mit dem Rhizom von Curcuma longa oder mit Auszügen von Codiaeum variegatum (Kroton). In langwierigen Gesängen ruft er dann alle Geister auf und zählt die Opfergaben auf, die für sie bereitliegen.

Die Hauptwaffe des Schamanen für den Schutz vor Angriffen gefährlicher Geister ist sein „Blattschwert“, ein Blatt der Keulenlilie, das mit ausgeschnittenen und mit Zierknoten ausgestatteten jungen Blättern der Kokospalme dekoriert ist. Auch Krone und Manschetten des Schamanen sind aus solchen Kokosblättern gefertigt.

\section{Das spirituelle Verbindungsglied des Schamanen zur Geisterwelt}

Mit dem Blattschwert in der Hand umtanzt der Schamane gegen den Uhrzeigersinn ein von der Decke herabhängendes weißes oder gemustertes Tuch. Im oberen Bereich ist es rundherum von herabhängenden jungen Betelnusspalmen-Blütenständen umgeben. Dazu kommen Verzierungen aus Palmblättern. Dies ist das heiligste Attribut der Zeremonie. Es ist das Verbindungsglied des medialen Schamanen zur Geisterwelt und umgekehrt. Im obersten Bereich ist ein hölzernes Kreuz innerhalb eines Ringes verborgen. Es symbolisiert die Wegkreuzung, die den Schamanen spirituell zu den acht Wegen oder Ebenen in der Geisterwelt führt, an denen die Geisterfamilien in ihren Dörfern leben.

Nach und nach werden alle Opfergaben gebracht und in devoter Form den Geistern angeboten. Hier sucht der Schamane auch die Erleuchtung zur Identifizierung des gesuchten Schadgeistes. Schälchen enthalten zur Erkennt-

Abb. 4 (oben): Mit Reishäufchen in den fünf aus Pflanzen gewonnenen Farben Schwarz, Rot, Gelb, Grün und Weiß auf den Opferplattformen vor dem Haus sollen die Geister herangelockt werden.

Abb. 5 (Mitte): Im Raum sollen sich die Geister auf kleinen Schaukeln niederlassen.

Abb. 6 (unten): Heranlocken der Geister aus den vier Himmelsrichtungen mit den vier hundeartigen Figuren und gefärbtem Wasser. Rechts gefärbter Reis geformt zu fünf Herzen. 
nis-Gewinnung u.a. kleine BienenwachsFackeln, einen Spiegel, Reis und Zweige von Kroton mit seinen dunkelgrünen, gelb gesprenkelten Blättern. Diese Pflanze hat eine magische Bedeutung, weil sie als unsterblich gilt und aussieht, als ob sie mit gelbem, heiligen Reis bestreut wäre. Alle Aktionen des Schamanen werden dröhnend von mehreren Trommeln, großen und sechs kleinen Gongs, mit denen die Melodie vorgegeben wird, begleitet. Melodie und Rythmus variieren je nach Tätigkeit des Schamanen.

\section{Opfergaben, Lockspeisen und Ersatzseelen für die Geister}

Die wichtigste Lockspeise für die Geister ist gekochter Reis in den Farben Schwarz, Rot, Gelb, Grün und Weiß. Die schwarze Farbe wird aus den Blättern von Archidendron gewonnen. Rot wird aus den Früchten von Bixa orellana extrahiert. Gelb liefern Kroton oder Kurkuma. Grün wird aus den Blättern von Jasminum sambac und Cordyline fruticosa gewonnen. Um die Geister von draußen heranzulocken, werden schon vor dem Eingang kleine Plattformen aus MelicopeHolz errichtet und mit Reishäufchen in den fünf Farben belegt. In der Mitte des Tellers mit dem gefärbten Reis befinden sich zusätzlich rote Blütenblätter. Die Eckpfosten der linken Plattform sind oben wie die Hüte von Darstellungen böser Geister angespitzt. Um die große Zahl von Geistern zu befriedigen, werden im Innenraum an den Wänden Reihen von Miniaturschaukeln mit vielen "Sitzebenen“ aufgehängt. Auf jeder Ebene liegen auf einem Stück eines Bananenblattes die kleinen Häufchen Reis in den fünf Farben. Manche Schamanen bieten die Farben

Abb. 7 (oben): Ein Geistersitz mit Miniaturopfergaben in Form von Gong und Schmiede-Blasebalg.

Abb. 8 (Mitte): Ein Haufen Zweige von Ritualpflanzen, die als Geistersitze angenommen werden, liegen vor dem Eingang. Der Schamane versucht mit Fackelbeleuchtung und in devoter Haltung singend die darin verborgenen Geister in den Raum zu locken.

Abb. 9 (unten): Nachdem die Zweige zum Altar gebracht wurden, werden die Geister gebeten, sich im Altarbereich niederzulassen.
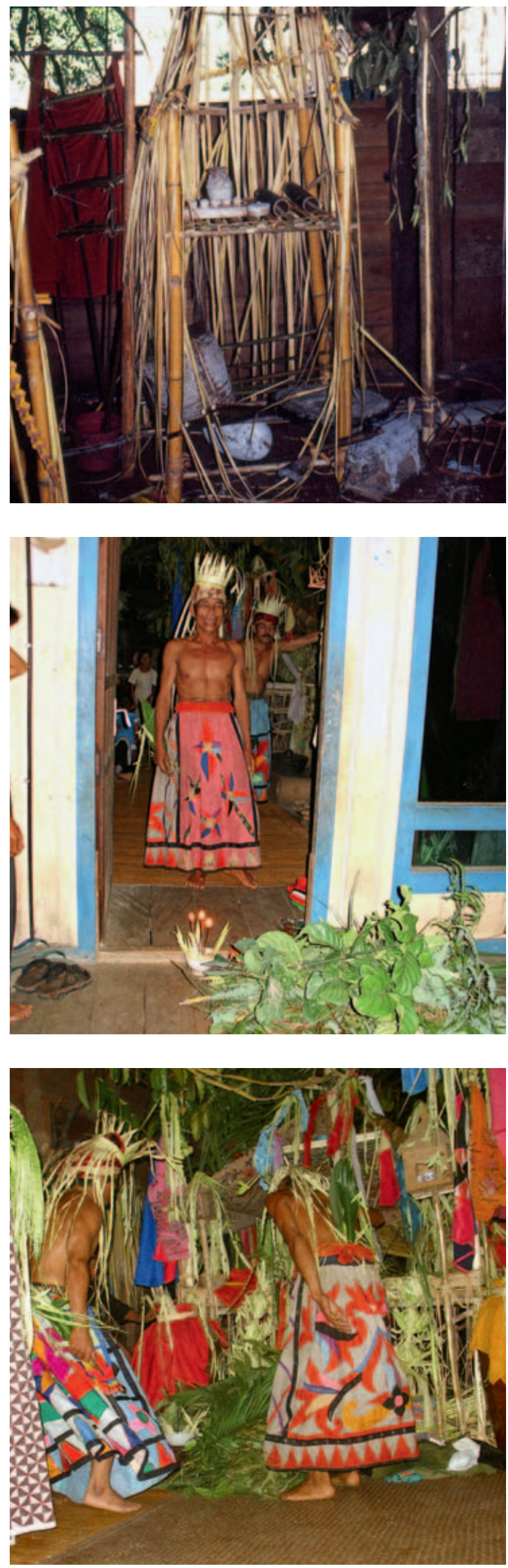

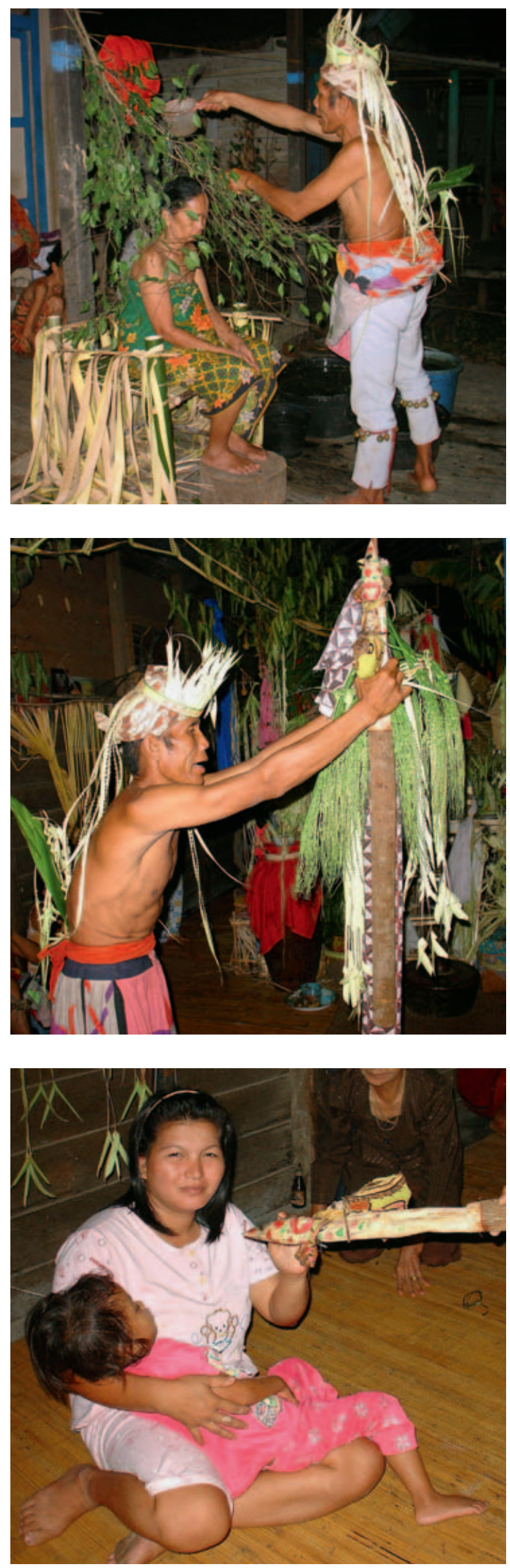

zusätzlich auch in flüssiger Form an. Weißer Reis in der Mitte ersetzt die fünfte Farbe. Die vier Gläschen sind von vier hundeartigen Figuren aus dem Holz von Melicope flankiert. Hier sollen die Geister aus den vier Himmelsrichtungen herangelockt werden. Diese Vorstellung dürfte auch auf alt-hinduistischen Einfluss zurückgehen.

Die große hundeartige Figur stellt den Schutzgeist für den Schamanen dar. Sie ist fürsorglich mit einem Stoff, gefertigt aus den Blattfasern von Curculigo latifolia, bekleidet. Auf einem weiteren Teller befindet sich Reis in fünf Farben, der zu fünf Herzen geformt ist. Figuren und Statuen, die Geister darstellen, von Geistern besetzt werden oder sie anziehen sollen, sind grundsätzlich aus dem Holz von Melicope incana gefertigt. Sie haben oft menschenähnliche Gestalt. Ein besonders mächtiger und böser Geist ist Banci. Ihm werden im Freien in einer großen bootsförmigen Konstruktion die Speisen und Lebensmittel geopfert, die die Menschen schätzen.

\section{Hereinlocken der Geister und die Diagnose}

Um den Schadgeist identifizieren zu können, hört sich der Schamane die langatmigen Krankengeschichten von den Familienangehörigen der Patienten einschließlich ihrer Träume an. Dann müssen alle Geister in den Raum gelockt werden. Sie sollen sich im Altarbereich und beim gefärbten Reis in den Miniaturschaukeln niederlassen. Dies erfolgt in der 2. und 3. Nacht des viernächtigen Rituals. Um den Geistern das Hereinkommen zu erleichtern, ist die Spitze des

Abb. 10 (oben): Der Schamane gießt Weihwasser über einen Ast von Ficus benjamina auf eine Patientin.

Abb. 11 (Mitte): Der identifizierte Schadgeist wird in einer "Spuckstatue“ dargestellt. Die Statue ist bemalt und mit Blüten von Basilikum geschmückt. Der Schamane hat der Figur ein bemaltes Beutelchen um den Hals gehängt, in dem der Krankheitsstoff wieder in das Geisterreich zurückgebracht werden soll.

Abb. 12 (unten): Der Krankheitsstoff kann direkt mit dem eigenen Speichel an den Schadgeist in der "Spuckstatue“ zurückgegeben werden. Die Mutter klebt Speichel des erkrankten Kindes an die Statue. 
Altars in der zweiten Nacht durch ein langes Rotang-Stück mit der äußersten Opferplattform vor dem Haus verbunden. In der dritten Nacht kommt noch eine leiterartige Verbindung hinzu. Auch ein Haufen großer Zweige aus dem Wald, der den Geistern als Wohnstätte dienen soll, liegt in der dritten Nacht vor dem Eingang. Er besteht u. a. aus den acht Arten, die rund um den Altar verteilt wurden. Einladende kleine Fackeln werden in den Eingang gestellt und der Schamane erscheint hier in devoter, höflicher Haltung und lädt die Geister singend ein, hineinzukommen. Die Zweige werden hereingeholt. Wenn sich die Geister im Altarbereich niedergelassen haben, werden die Zweige wieder hinaus gebracht. Es muss jetzt im Raum von Geistern wimmeln. In der dritten Nacht muss der die Krankheit(en) verursachende Geist identifiziert sein.

\section{Die rituelle Prophylaxe}

Um zu verhindern, dass die noch gesunde Verwandtschaft der Patienten ebenfalls erkrankt, führen die Schamanen prophylaktische Rituale durch. Alle Anwesenden werden auf der Stirne mit Reispaste (und mit Blut der geopferten Tiere) beschmiert und mit Weihwasser besprengt. Der Schamane hält das böse Geister abwehrende Blattschwert sowie Fackeln und bunte Tücher über ihre Köpfe. Vor dem Haus wird ein thronartiger Sitz errichtet, auf dem Familienangehörige der Patienten Platz nehmen. Der Schamane hält einen großen Ast von Ficus benjamina über ihren Kopf und gießt ausgiebige Mengen Weihwasser über Zweig und Mensch. Ficus benjami$n a$ ist der Sitz der Baumgeister, die die Lebenslänge beeinflussen können, Irrsinn verursachen, aber auch heilen können. Hier ersucht sie der singende Schamane, ihren positiven Einfluss auf die Person auszuüben.

Abb. 13 (oben): Der Krankheitsstoff kann durch intensiven Kontakt dem Beutel der Geisterstatue übergeben werden.

Abb. 14 (Mitte): Eine Seele wird in einem Geisterhäuschen am Altar lokalisiert.

Abb. 15 (unten): Die fadenförmige Körperteilseele wird dem Patienten in den erkrankten Körperteil einmassiert.
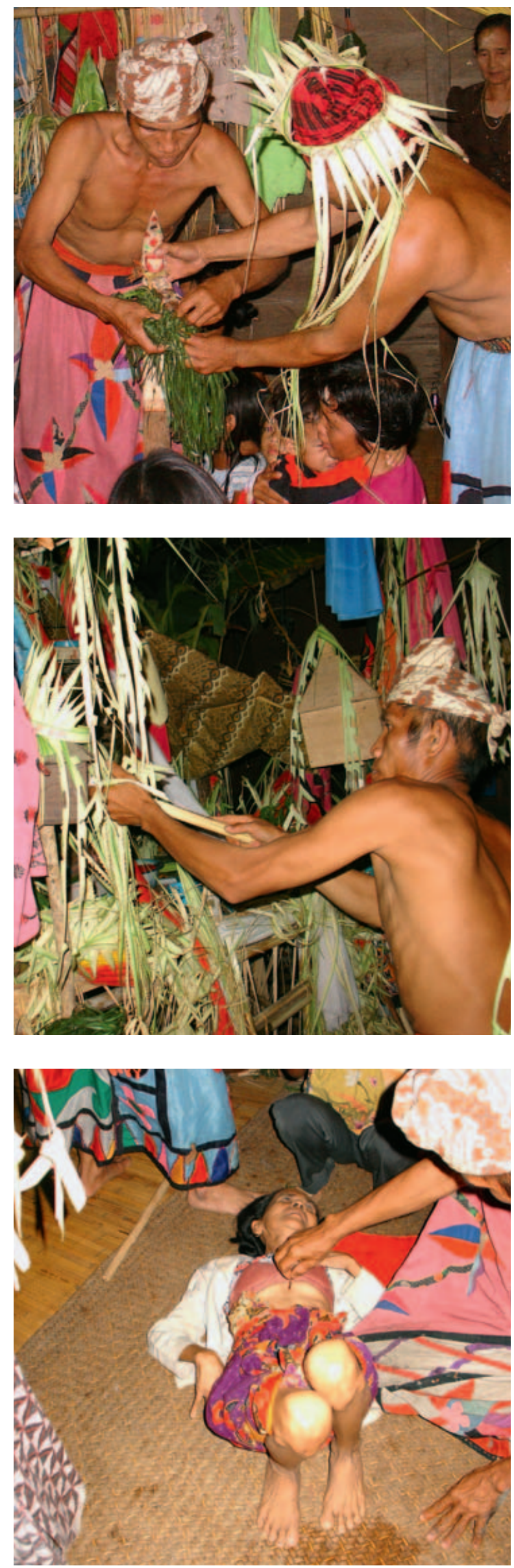


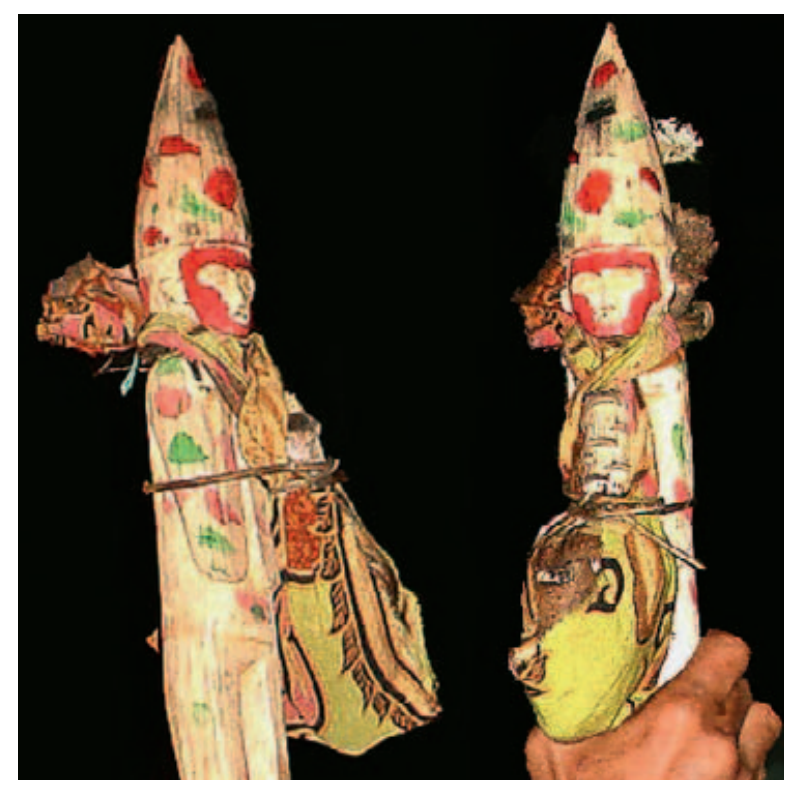

\section{Die rituelle Therapie}

Der Schamane legt ein quastenartig zusammengebundenes Bündel eines aufgespleißten Bananenblattes singend dem Patienten auf den erkrankten Körperteil und extrahiert damit den Krankheitsstoff. Er schüttelt oder pflückt diesen heraus und übergibt ihn dem Schälchen mit dem Opferblut oder dem bunten Reis. Wenn der Schadgeist identifiziert und willig ist, wird ihm der Krankheitsstoff persönlich übergeben. Ob er willig ist, erkennt der Schamane an bestimmten Zeichen an der Leber und der Milz des geopferten Schweines. Die Übergabe erfolgt mittels einer Spuckstatue. Das ist eine aus Melicope incana geschnitzte Figur, die den Schadgeist darstellt. In einem bemalten Beutel soll der Geist den Krankeitsstoff wieder in sein Geisterreich zurückbringen. Dieser Beutel besteht aus dem ersten Deckblatt am Blütenstand der Pinang-Palme. Kranke können ihren Krankheitsstoff an die Statue spucken oder mit eigenem Speichel daran schmieren und damit los werden, oder die Mutter macht dies mit dem Speichel des kranken Kindes. Die Statue mit dem Krankheitsstoff wird anschließend in den Wald zurückgebracht (vgl. auch ZAHORKA 2002).

Im letzten Abschnitt eines Rituals tanzt sich der Schamane bei furioser Trommelmusik in Trance, fällt hin, und wird mit einem Tuch zugedeckt. In diesem Zustand erfährt er, wo der Schadgeist die entwendete Körperteilseele de- poniert hat. Wenn er nach etwa einer halben Minute wieder zu sich kommt, ergreift er gierig ein mit gekochtem Reis ausgefülltes Bambusinternodium und stochert damit fieberhaft in die Stelle hinein, die ihm eingegeben wurde. Das kann z. B. das Geäst einer Pflanze oder eines der Geisterhäuschen am Altar sein. Aus dem vorderen Ende des Bambusabschnittes zieht er dann die fadenförmige Seele heraus, betrachtet sie, um herauszufinden, zu welchem Patienten sie gehört, und massiert sie dann diesem Patienten in den kranken Körperteil ein. Dies ist das Ende der Therapie. Mit einem nächtlichen Mahl der geopferten Schweine oder Hühner endet das Heilungsritual. Die rechte Körperhälfte der Opfertiere erhalten die Schamanen und ihre Helfer.

Die Benuaq nennen ihre Weltanschauung adat nahaa. Die indonesische offizielle Bezeichnung für diese Stammesreligion ist HinduKaharingan. Diese Bezeichnung kennen die Benuaq aber nicht. Ihre Nähe zum Hinduismus zeigt sich jedoch auch in ihrem Glauben an einen Schöpfergott Letala (abgeleitet von alt-hinduistischen Schöpfergott Batara?) und an die gottgesandten Geister.

\section{Dank}

Für Hinweise zur Pflanzenidentifizierung danke ich Dr. Christian Gönner und Anatolius Teng und vom Herbarium Bogoriense Dr.-Ing. Harry Wiriadinata, Dr.-Ing. Yohannes Purwanto, Ary P. Keim und Dr. Johannis P. Mogea.

\section{Literatur}

Gönner, C. 2002: A forest tribe of Borneo. - New Delhi. ZaHorka, H. 2002: A wooden figure used to take on human diseases - Shamanistic healing rituals of the Basap in east Kalimantan. - Borneo Res. Bull. 33: 181-183.

Abb. 16: Die Spuckstatue des krankmachenden Geistes mit dem umgehängten Beutel. 